\title{
Bone responsiveness to parathyroid hormone is negatively associated with parathyroid hormone-lowering drug use in patients undergoing hemodialysis: a cross- sectional study
}

Naoto Tominaga ${ }^{1,2^{*}}$, Tomoki Yonaha ${ }^{3}$, Masayuki Yamanouchi ${ }^{4}$, Hirofumi Sumi ${ }^{1,2}$, Yasuhiro Taki ${ }^{1,2}$, Yugo Shibagaki ${ }^{1}$, Kazuhiro Shiizaki ${ }^{5}$ and Shozo Yano ${ }^{6}$

\begin{abstract}
Background: Parathyroid hormone (PTH) acts on bone to indirectly increase the number and activity of osteoclasts. Thus, PTH has a stimulatory effect on bone resorption and upregulates bone turnover. However, the responsiveness of bone to PTH varies widely among patients receiving dialysis. In fact, relative to the serum PTH level, the level of serum tartrate-resistant acid phosphatase-5b (TRACP-5b), a bone resorption marker derived from osteoclasts, varies as well. This study aimed to examine factors related to bone responsiveness to PTH in patients undergoing chronic hemodialysis (HD).

Methods: This study included patients receiving chronic HD in Kawasaki Municipal Tama Hospital (Kanagawa, Japan) and Yonaha Medical Clinic (Okinawa, Japan) and excluded patients who received HD for less than 6 months, those who received a combination of $\mathrm{HD}$ and peritoneal dialysis, and those who had cancer bone metastases or myeloma. The TRACP-5b/intact PTH (iPTH) ratio was created as an index of bone responsiveness to PTH, categorized into tertiles (low, medium, and high), and a cross-sectional study was conducted. $P<0.05$ indicated statistically significant differences.
\end{abstract}

\footnotetext{
* Correspondence: tominaga1213@kbd.biglobe.ne.jp

${ }^{1}$ Division of Nephrology and Hypertension, Department of Internal Medicine,

St. Marianna University School of Medicine, 2-16-1, Sugao, Miyamae-ku,

Kawasaki, Kanagawa 216-8511, Japan

${ }^{2}$ Division of Nephrology and Hypertension, Kawasaki Municipal Tama

Hospital, Kawasaki, Kanagawa, Japan

Full list of author information is available at the end of the article
}

C The Author(s). 2021 Open Access This article is licensed under a Creative Commons Attribution 4.0 International License, which permits use, sharing, adaptation, distribution and reproduction in any medium or format, as long as you give appropriate credit to the original author(s) and the source, provide a link to the Creative Commons licence, and indicate if changes were made. The images or other third party material in this article are included in the article's Creative Commons licence, unless indicated otherwise in a credit line to the material. If material is not included in the article's Creative Commons licence and your intended use is not permitted by statutory regulation or exceeds the permitted use, you will need to obtain permission directly from the copyright holder. To view a copy of this licence, visit http://creativecommons.org/licenses/by/4.0/. The Creative Commons Public Domain Dedication waiver (http://creativecommons.org/publicdomain/zero/1.0/) applies to the data made available in this article, unless otherwise stated in a credit line to the data. 
Results: One hundred and six patients were analyzed. Age $(P=0.010)$, body mass index (BMI) $(P=0.003)$, use of calcium-sensing receptor (CaSR) agonists $(P=0.008)$, use of vitamin $D$ receptor activators (VDRAs) $(P=0.012)$, plasma iPTH level $(P<0.001)$, serum $1,25(\mathrm{OH})_{2} \mathrm{D}$ level $(P=0.003)$, and serum TRACP-5b level $(P<0.001)$ were significantly different among the three categories. In the single linear regression analysis, age $(P=0.016)$, corrected serum calcium level $(P=0.007)$, and In $\left[1,25(\mathrm{OH})_{2} \mathrm{D}\right](P=0.044)$ showed a significant positive correlation with In $[T R A C P-5 \mathrm{~b} /$ iPTH], whereas BMI $(P=0.026)$, use of CaSR agonists $(P=0.001)$, use of VDRAs $(P=0.009)$, and serum phosphorus level $(P=0.018)$ showed a significant negative correlation. Upon conducting multiple linear regression analysis incorporating significant variables in the single linear regression analysis, a significant negative correlation was observed between the TRACP-5b/iPTH ratio and intravenous administration of a CaSR agonist (etelcalcetide) and/or a VDRA (calcitriol or maxacalcitol) in all the adjusted models.

Conclusions: Bone responsiveness to PTH is negatively correlated with the intravenous administration of a CaSR agonist and/or a VDRA in patients undergoing chronic HD.

Keywords: Hemodialysis, Parathyroid hormone, Tartrate-resistant acid phosphatase-5b, Calcium-sensing receptor agonists, Vitamin D receptor activators

\section{Background}

High bone turnover is the main pathophysiology of secondary hyperparathyroidism (SHPT) in patients undergoing chronic hemodialysis (HD) and leads to a high risk of fracture [1]. A report from the Dialysis Outcomes and Practice Patterns Study showed that the incidence of fractures was significantly higher in patients on chronic HD than that in the general population, with chronic HD patients showing a 3.7-fold higher unadjusted relative risk of death [2]. Parathyroid hormone $(\mathrm{PTH})$ is one of the promoting factors for bone turnover. However, bone responsiveness to PTH varies from individual to individual. For example, African Americans show lower bone responsiveness to PTH than Caucasians; therefore, African Americans may require a higher PTH level to maintain the same degree of bone turnover as Caucasians [3].

PTH stimulates osteoblasts to secrete receptor activator of nuclear factor-kappa B ligand (RANKL), which then stimulates the osteoclast precursors to mature [4]. Mature osteoclasts produce tartrate-resistant acid phosphatase 5b (TRACP-5b), which is released into the circulation as a specific biomarker of osteoclast number and activity [5]. PTH levels can now be controlled through treatment of SHPT. Particularly, calciumsensing receptor (CaSR) agonists and vitamin $\mathrm{D}$ receptor activators (VDRAs) are commonly used to control the high-turnover bone status in patients on chronic HD [6]. However, the association between the use of a CaSR agonist and/or a VDRA and bone responsiveness to PTH has not been reported in a population undergoing dialysis.

In the present study, we created the TRACP-5b/intact PTH (iPTH) ratio as an index of bone responsiveness to PTH, categorized into tertiles (low, medium, and high), and aimed to compare clinical and laboratory factors between the low, medium, and high TRACP-5b/iPTH ratio groups of patients undergoing chronic $\mathrm{HD}$. We further aimed to examine the association between the use of a CaSR agonist and/or a VDRA and the TRACP-5b/ iPTH ratio in outpatients undergoing chronic HD.

\section{Methods}

Patients and materials

Data for the present study were obtained from the pooled patient electronic health record system of the Kawasaki Municipal Tama Hospital in Kanagawa and Yonaha Medical Clinic in Okinawa. We retrospectively reviewed clinical and laboratory data from outpatients who had received chronic HD from April 2018 to September 2018. This study was conducted in accordance with the Declaration of Helsinki and was approved by the Institutional Committee on Human Research of St. Marianna University School of Medicine [the Institutional Review Board (IRB) at St. Marianna University School of Medicine (IRB approval number: 4291)]. The requirement for informed consent was waived due to the de-identified nature of the analyses.

\section{Study design and procedures}

This retrospective cross-sectional study was designed to investigate the association between CaSR agonist and/or VDRA use (as a binary variable) and the TRACP-5b/ iPTH ratio (as a continuous variable) among patients undergoing chronic HD. Patients who had been undergoing chronic HD for less than 6 months and those undergoing combined $\mathrm{HD}$ and peritoneal dialysis were excluded. Patients with bone metastases and/or multiple myeloma, which have potential effects on bone responsiveness to PTH, were also excluded.

We utilized the means of 11 consecutive values for serum albumin (Alb), calcium $(\mathrm{Ca})$, and phosphorus $(\mathrm{P})$ 
levels measured in the blood twice per month as well as the means of six consecutive values for the plasma intact parathyroid hormone (iPTH) level measured once per month prior to obtaining serum bone-specific alkaline phosphatase (BAP), TRACP-5b, 25-hydroxyvitamin D [25(OH)D], and 1,25-dihydroxycholecalciferol $\left[1,25(\mathrm{OH})_{2} \mathrm{D}\right]$ level data as of September 2018. We used Payne's formula to correct the serum $\mathrm{Ca}$ (cCa) level based on the serum Alb level [7]. The serum BAP level was measured using the chemiluminescent enzyme immunoassay (CLEIA) (LSI Medience Corporation, Chiyoda-ku, Tokyo, Japan), the serum TRACP-5b level was measured using the enzyme immunoassay (EIA) (LSI Medience Corporation), the plasma iPTH level was measured using the electrochemiluminescence immunoassay (ECLIA) (LSI Medience Corporation), the serum $25(\mathrm{OH}) \mathrm{D}$ level was measured using the chemiluminescence immunoassay (CLIA) (LSI Medience Corporation), and the serum $1,25(\mathrm{OH})_{2} \mathrm{D}$ level was measured using double-antibody radioimmunoassay (RIA) (LSI Medience Corporation). All blood tests were performed prior to HD, in the first HD session of the week. Patient exposure to clinical variables of interest was defined by data regarding duration of chronic HD, type of anticoagulant used during HD, disease diagnosis (i.e., diabetes, ischemic heart disease, or cerebrovascular disease), drug prescription [i.e., CaSR agonist (cinacalcet/evocalcet, etelcalcetide), VDRA (alfacalcidol/falecalcitriol, calcitriol, maxacalcitol), P binder (Ca containing, Non-Ca containing, both), steroid, $\beta$-blocker, statin, warfarin, or bisphosphonate], or history of fracture.

A recent study reported the non-inferiority of evocalcet to cinacalcet in suppressing the iPTH level by acting as an oral allosteric modulator of CaSR [8]; therefore, patients on evocalcet were grouped with the patients on cinacalcet. Etelcalcetide is an intravenous formula of a direct activator of CaSR; therefore, it was considered in a separate group although the number of patients using the drug was small. A patient receiving falecalcitriol was grouped with the patients receiving alfacalcidol, since both drugs are oral VDRAs. Data regarding the anklebrachial index (ABI) were used, as peripheral artery disease is associated with low bone turnover in prevalent nondiabetic patients with end-stage renal disease (ESRD) [9]. The body mass index (BMI) was calculated by dividing weight $(\mathrm{kg})$ by height squared $\left(\mathrm{m}^{2}\right)$.

\section{Statistical analysis}

All data were summarized using descriptive statistics, such as median, interquartile range (IQR), frequency, and percentage. For continuous variables, the KruskalWallis test was used to assess the significance of differences in patient characteristics among the three TRACP-5b/iPTH ratio categories (low, medium, and high). For discrete variables, the chi-square test was used to compare data among the three groups. Single and multiple linear regression analyses were used to examine factors affecting the TRACP-5b/iPTH ratio as of September 2018. A multiple linear regression analysis was performed with adjustment for age, sex, BMI, and serum Alb level using variables identified as significant in the single linear regression analysis. In the single and multiple linear regression analyses, values of plasma iPTH, serum 25(OH)D, 1,25(OH $)_{2} \mathrm{D}, \quad B A P$ and TRACP-5b levels and the TRACP-5b/iPTH ratio were transformed to natural logarithms $(\ln )$ for their skewed distribution. Statistical analyses were performed using Stata version 14 (StataCorp LP, College Station, TX, USA). Statistical significance was defined at $P<0.05$.

\section{Results}

Patient characteristics

Median patient age, BMI, and duration of chronic HD were 68 years, $21.5 \mathrm{~kg} / \mathrm{m}^{2}$, and 7.9 years, respectively. The median serum Alb, cCa, P, BAP, and TRACP-5b levels were $3.6 \mathrm{~g} / \mathrm{dL}, 9.1 \mathrm{mg} / \mathrm{dL}, 5.4 \mathrm{mg} / \mathrm{dL}, 14.2 \mu \mathrm{g} / \mathrm{L}$, and $559 \mathrm{mU} / \mathrm{dL}$, respectively. The median plasma iPTH level was $199.1 \mathrm{pg} / \mathrm{mL}$. Among the 106 patients included, 45 were women $(42.5 \%), 38$ had diabetes (type 2 diabetes in all cases) (35.8\%), 61 had been prescribed CaSR agonist (57.5\%), and 97 had been prescribed VDRA (91.5\%). The causes of ESRD included diabetic nephropathy in 34 cases (32.1\%), nephrosclerosis in 25 cases (23.6\%), glomerulonephritis in 28 cases $(26.4 \%)$, and other causes in 19 cases $(17.9 \%)$ (Table 1$)$.

\section{Comparison of the three TRACP-5/iPTH ratio categories (low, medium, and high)}

We compared patient attributes and laboratory data among the low, medium, and high TRACP-5/iPTH ratio categories (Table 2, Fig. 1).

Median age, BMI, plasma iPTH, and serum $1,25(\mathrm{OH})_{2} \mathrm{D}$, TRACP-5b levels; the number of patients receiving CaSR agonists; and the number of patients receiving VDRAs were significantly different among the three categories (Table 2). The types of CaSR agonists and VDRAs used were also significantly different among the three categories.

\section{Correlation between plasma iPTH and serum TRACP-5b levels}

Relative to the serum iPTH level, the serum TRACP-5b level of the patients varied as well (adjusted $R^{2}=0.049$ ) (Fig. 2a). Serum TRACP-5b levels showed a weaker correlation with plasma iPTH levels in patients treated with the CaSR agonist than in patients treated without the CaSR agonist (Fig. 2b). Similarly, serum TRACP-5b levels showed a weaker correlation with plasma iPTH 
Table 1 Patient characteristics

\begin{tabular}{|c|c|}
\hline & $N=106$ \\
\hline Age, years & $68[60.75]$ \\
\hline Female sex, n (\%) & $45(42.5)$ \\
\hline $\mathrm{BMl}, \mathrm{kg} / \mathrm{m}^{2}$ & $21.5[19.6,24.3]$ \\
\hline Chronic HD vintage, years & $7.9[2.9,12.3]$ \\
\hline Anticoagulant during HD, n (\%) & $106(100)$ \\
\hline None & $0(0)$ \\
\hline Heparin & $90(84.9)$ \\
\hline LMWH & $10(9.4)$ \\
\hline Nafamostat & $6(5.7)$ \\
\hline Cause of ESRD, n (\%) & $106(100)$ \\
\hline Diabetic nephropathy & $34(32.1)$ \\
\hline Nephrosclerosis & $25(23.6)$ \\
\hline Glomerulonephritis & $28(26.4)$ \\
\hline Others & $19(17.9)$ \\
\hline Diabetes, n (\%) & $38(35.8)$ \\
\hline Ischemic heart disease, n (\%) & $27(25.5)$ \\
\hline Cerebrovascular disease, n (\%) & $18(17.0)$ \\
\hline CaSR agonist use, n (\%) & $61(57.5)$ \\
\hline None & $45(42.5)$ \\
\hline Cinacalcet/ Evocalcet & $55(51.9)$ \\
\hline Etelcalcetide (i.v.) & $6(5.7)$ \\
\hline VDRA use, n (\%) & $97(91.5)$ \\
\hline None & $9(8.5)$ \\
\hline Alfacalcidol/ Falecalcitriol & $48(45.3)$ \\
\hline Calcitriol (i.v.) & $32(30.2)$ \\
\hline Maxacalcitol (i.v.) & $17(16.0)$ \\
\hline P binder use, n (\%) & $90(84.9)$ \\
\hline None & $16(15.1)$ \\
\hline Ca containing & $12(11.3)$ \\
\hline Non-Ca containing & $55(51.9)$ \\
\hline Both & $23(21.7)$ \\
\hline Steroid use history, n (\%) & $13(12.3)$ \\
\hline$\beta$ blocker use, n (\%) & $48,(45.3)$ \\
\hline Statin use, n (\%) & $28(26.4)$ \\
\hline Warfarin use, n (\%) & $7(6.6)$ \\
\hline Bisphosphonate use, n (\%) & $14(13.2)$ \\
\hline Fracture history, n (\%) & $15(14.2)$ \\
\hline Ankle-brachial index & $1.10[1.03,1.18]$ \\
\hline Alb, g/dL & $3.6[3.4,3.8]$ \\
\hline $\mathrm{cCa}, \mathrm{mg} / \mathrm{dL}$ & $9.1[8.5,9.4]$ \\
\hline $\mathrm{P}, \mathrm{mg} / \mathrm{dL}$ & $5.4[4.5,6.0]$ \\
\hline iPTH, pg/mL & $199.1[119.0,277.2]$ \\
\hline 25(OH)D, ng/mL & $14.3[11.0,18.9]$ \\
\hline $1,25(\mathrm{OH})_{2} \mathrm{D}, \mathrm{pg} / \mathrm{mL}$ & $13[8,19]$ \\
\hline
\end{tabular}

Table 1 Patient characteristics (Continued)

\begin{tabular}{ll}
\hline & $\boldsymbol{N}=\mathbf{1 0 6}$ \\
\hline BAP, $\mu \mathrm{g} / \mathrm{L}$ & $14.2[10.8,18.3]$ \\
TRACP-5b, mU/dL & $559[339,833]$ \\
\hline
\end{tabular}

Abbreviations: Alb Serum albumin, BAP Bone-specific alkaline phosphatase, BMI Body mass index, CaSR Calcium-sensing receptor, cCa Corrected Ca, ESRD Endstage renal disease, HD Hemodialysis, iPTH Intact parathyroid hormone, i.v. Intravenous, LMWH Low-molecular-weight heparin, $P$ Phosphorus, TRACP-5b Tartrate-resistant acid phosphatase-5b, VDRA Vitamin D receptor activator Values are expressed as the median [interquartile range (IQR)] or as frequencies and percentages [n (\%)]

levels in patients treated with the VDRA than in patients treated without the VDRA (Fig. 2c).

\section{Association between CaSR agonists and VDRAs and the TRACP-5b/iPTH ratio}

We examined whether CaSR agonist and/or VDRA use was associated with bone responsiveness to PTH. The results of the single linear regression analysis using $\ln$ [TRACP-5b/iPTH] as a dependent variable are shown in Table 3. The single linear regression analysis revealed a significant positive correlation between ln [TRACP-5b/ iPTH $]$ and age, serum cCa level, and $\ln \left[1,25(\mathrm{OH})_{2} \mathrm{D}\right]$ and a significant negative correlation between $\ln$ [TRACP-5b/iPTH] and BMI, serum P level, CaSR agonist use, and VDRA use. Moreover, upon dividing the patient cohort according to the types of CaSR agonists and VDRAs, the single linear regression analysis also showed a significant negative correlation between In [TRACP$5 \mathrm{~b} / \mathrm{iPTH}]$ and cinacalcet/evocalcet use, etelcalcetide use (intravenously, i.v.), calcitriol use (i.v.), and maxacalcitol use (i.v.).

We next conducted a multiple linear regression analysis with adjustment for age, sex, BMI, and serum Alb level using variables identified as significant in the single linear regression analysis (i.e., CaSR agonist use, VDRA use, serum $\mathrm{cCa}$ level, serum $\mathrm{P}$ level, and $\mathrm{ln}$ $\left.\left[1,25(\mathrm{OH})_{2} \mathrm{D}\right]\right)($ Table 4). Model 1 was unadjusted; model 2 was adjusted for age and sex; model 3 was adjusted for age, sex, and BMI; and model 4 was adjusted for age, sex, BMI, and serum Alb level. In all adjusted models, etelcalcetide use (i.v.), calcitriol use (i.v.), and maxacalcitol use (i.v.) exhibited a significant negative correlation with $\ln$ [TRACP-5b/iPTH].

Additionally, in order to uncover the association between mineral metabolism and $\ln$ [TRACP-5b/iPTH], we performed an additional multiple linear regression analysis for $\ln$ [TRACP-5b/iPTH] only using variables identified as significant in the single linear regression analyses, such as age, BMI, serum cCa, P levels, and $\ln$ $\left[1,25(\mathrm{OH})_{2} \mathrm{D}\right]$, shown in Table 3 . Only the serum $\mathrm{cCa}$ level showed a significant positive association with $\ln$ [TRACP-5b/iPTH] (Table 5). 
Table 2 Comparison of the TRACP-5b/iPTH ratio among the three categories (low, medium, and high)

\begin{tabular}{|c|c|c|c|c|}
\hline & \multicolumn{3}{|c|}{ TRACP-5b/iPTH ratio } & \multirow[t]{3}{*}{$P$ value } \\
\hline & $\begin{array}{l}\text { Low } \\
\text { (Quartile 1) }\end{array}$ & $\begin{array}{l}\text { Medium } \\
\text { (Quartile 2) }\end{array}$ & $\begin{array}{l}\text { High } \\
\text { (Quartile 3) }\end{array}$ & \\
\hline & $<2.06$ & $\geqq 2.06,<4.46$ & $4.46 \leqq$ & \\
\hline N & 36 & 35 & 35 & - \\
\hline Age, years & $65[54,72]$ & $68[63,75]$ & $69[64,76]$ & 0.010 \\
\hline Female sex, $n$ & 13 & 15 & 17 & 0.568 \\
\hline $\mathrm{BMI}, \mathrm{kg} / \mathrm{m}^{2}$ & $21.9[20.6,24.6]$ & $23.0[20.1,27.1]$ & $20.1[18.4,21.8]$ & 0.003 \\
\hline Chronic HD vintage, years & $6.8[3.6,13.5]$ & $8.7[3.0,12.3]$ & $8.0[2.5,12.0]$ & 0.730 \\
\hline \multicolumn{5}{|c|}{ Anticoagulant during HD, n (\%) } \\
\hline Heparin & $30(83.3)$ & 31 (88.6) & $29(82.9)$ & \multirow[t]{3}{*}{0.299} \\
\hline LMWH & $4(11.1)$ & $4(11.4)$ & $2(5.7)$ & \\
\hline Nafamostat & $2(5.6)$ & $0(0)$ & $4(11.4)$ & \\
\hline \multicolumn{5}{|l|}{ Cause of ESRD, $n$} \\
\hline Diabetic nephropathy & 12 & 9 & 13 & \multirow[t]{4}{*}{0.840} \\
\hline Nephrosclerosis & 9 & 9 & 7 & \\
\hline Glomerulonephritis & 10 & 11 & 7 & \\
\hline Others & 5 & 6 & 8 & \\
\hline Diabetes, $\mathrm{n}$ & 15 & 10 & 13 & 0.506 \\
\hline Ischemic heart disease, $n$ & 10 & 9 & 8 & 0.892 \\
\hline Cerebrovascular disease, $\mathrm{n}$ & 9 & 4 & 5 & 0.274 \\
\hline CaSR agonist use, n (\%) & $26(72.2)$ & $22(62.9)$ & $13(37.1)$ & 0.008 \\
\hline None & $10(27.8)$ & $13(37.1)$ & $22(62.9)$ & \multirow[t]{3}{*}{0.001} \\
\hline Cinacalcet/ Evocalcet & $20(55.6)$ & $22(62.9)$ & $13(37.1)$ & \\
\hline Etelcalcetide (i.v.) & $6(16.7)$ & $0(0)$ & $0(0)$ & \\
\hline VDRA use, n (\%) & $35(97.2)$ & $34(97.1)$ & $28(80.0)$ & 0.012 \\
\hline None & $1(2.8)$ & $1(2.9)$ & $7(20.0)$ & \multirow[t]{4}{*}{0.001} \\
\hline Alfacalcidol/ Falecalcitriol & $10(27.8)$ & $18(51.4)$ & $20(57.1)$ & \\
\hline Calcitriol (i.v.) & $14(38.9)$ & $13(37.1)$ & $5(14.3)$ & \\
\hline Maxacalcitol (i.v.) & $11(30.6)$ & $3(8.6)$ & $3(8.6)$ & \\
\hline $\mathrm{P}$ binder use, $\mathrm{n}(\%)$ & $30(83.3)$ & 31 (88.6) & $29(82.9)$ & 0.759 \\
\hline None & $6(16.7)$ & $4(11.4)$ & $6(17.1)$ & \multirow[t]{4}{*}{0.471} \\
\hline Ca containing & $3(8.3)$ & $3(8.6)$ & $6(17.1)$ & \\
\hline Non-Ca containing & $20(55.6)$ & $22(62.9)$ & $13(37.1)$ & \\
\hline Both & $7(19.4)$ & $6(17.1)$ & $10(28.6)$ & \\
\hline Steroid use history, n (\%) & $5(13.9)$ & $4(11.4)$ & $4(11.4)$ & 0.935 \\
\hline$\beta$ blocker use, n (\%) & $18(50.0)$ & $18(51.4)$ & $12(34.3)$ & 0.277 \\
\hline Statin use, n (\%) & $12(33.3)$ & $7(20.0)$ & $9(25.7)$ & 0.441 \\
\hline Warfarin use, n (\%) & $0(0)$ & $4(11.4)$ & $3(8.6)$ & 0.130 \\
\hline Bisphosphonate use, n (\%) & $5(13.9)$ & $4(11.4)$ & $5(14.3)$ & 0.929 \\
\hline Fracture history, n & 3 & 6 & 6 & 0.468 \\
\hline Ankle-brachial index & $1.10[1.03,1.18]$ & $1.09[1.03,1.18]$ & $1.10[1.01,1.17]$ & 0.819 \\
\hline Alb, g/dL & $3.6[3.3,3.8]$ & $3.6[3.4,3.8]$ & $3.6[3.4,3.7]$ & 0.628 \\
\hline $\mathrm{cCa}, \mathrm{mg} / \mathrm{dL}$ & $8.9[8.4,9.3]$ & $9.2[8.6,9.6]$ & $9.1[8.7,9.5]$ & 0.109 \\
\hline$P, \mathrm{mg} / \mathrm{dL}$ & $5.6[4.7,6.4]$ & $5.3[4.5,6.0]$ & $5.0[4.3,5.6]$ & 0.054 \\
\hline iPTH, pg/mL & $300.1[227.9,441.2]$ & $196.7[129.3,269.2]$ & $118.8[66.8,187.8]$ & 0.0001 \\
\hline
\end{tabular}


Table 2 Comparison of the TRACP-5b/iPTH ratio among the three categories (low, medium, and high) (Continued)

\begin{tabular}{|c|c|c|c|c|}
\hline & \multicolumn{3}{|c|}{ TRACP-5b/iPTH ratio } & \multirow[t]{3}{*}{$P$ value } \\
\hline & $\begin{array}{l}\text { Low } \\
\text { (Quartile 1) }\end{array}$ & $\begin{array}{l}\text { Medium } \\
\text { (Quartile 2) }\end{array}$ & $\begin{array}{l}\text { High } \\
\text { (Quartile 3) }\end{array}$ & \\
\hline & $<2.06$ & $\geqq 2.06,<4.46$ & $4.46 \leqq$ & \\
\hline 25(OH)D, ng/mL & $14.0[11.6,18.0]$ & $16.6[12.3,20.3]$ & $13.3[9.7,17.3]$ & 0.075 \\
\hline $1,25(\mathrm{OH})_{2} \mathrm{D}, \mathrm{pg} / \mathrm{mL}$ & $10.0[5.0,14.5]$ & $15.0[11.0,22.0]$ & $16.0[8.0,21.0]$ & 0.003 \\
\hline $\mathrm{BAP}, \mu \mathrm{g} / \mathrm{L}$ & $12.7[9.3,18.2]$ & $14.3[10.8,18.3]$ & $15.0[11.3,19.4]$ & 0.343 \\
\hline TRACP-5b, mU/dL & $347.5[239.5,500.5]$ & $591.0[339.0,777.0]$ & $848.0[567.0,1060.0]$ & 0.0001 \\
\hline
\end{tabular}

Abbreviations: Alb Serum albumin, BAP Bone-specific alkaline phosphatase, BMI Body mass index, CaSR Calcium-sensing receptor, $c C a$ Corrected Ca, ESRD End-stage renal disease, $H D$ Hemodialysis, $i P T H$ Intact parathyroid hormone, i.v. Intravenous, LMWH Low-molecular-weight heparin, $P$ Phosphorus, TRACP-5b Tartrate-resistant acid phosphatase-5b, VDRA Vitamin D receptor activator

Values are expressed as the median [interquartile range (IQR)] or as frequencies and percentages [n (\%)]

\section{Discussion}

To the best of our knowledge, this study is the first report demonstrating that bone responsiveness to PTH is significantly negatively associated with the intravenous administration of CaSR agonist and/or VDRA in patients undergoing chronic HD. High bone turnover is the central pathophysiology in SHPT, which is related to an increased risk of fracture [1]. Therefore, the management of SHPT is expected to improve bone metabolism and reduce the risk of fractures. In this aspect, the management goal of iPTH levels remains an important issue. Based on the relationship between the iPTH level and mortality risk in observational studies [10], the Kidney Disease: Improving Global Outcomes (KDIGO) guideline for CKD-MBD in 2009 suggested revising the management goal of iPTH levels to 2-9 times $(130-585 \mathrm{pg} /$ $\mathrm{mL}$ ) of the normal upper limits [11]. Although the 2012 guidelines of the Japanese Society for Dialysis Therapy (JSDT) also suggested target iPTH levels of $60-240 \mathrm{pg} /$
$\mathrm{mL}$ based on mortality [12], the target levels of JSDT are close to the target iPTH levels of $150-300 \mathrm{pg} / \mathrm{mL}$ proposed by the K/DOQI guidelines in 2003 based on the correlation of iPTH levels with bone turnover by bone morphometry [13].

Bone loss observed in patients receiving chronic HD affected cortical bone mineral density and thickness, which are correlated with high PTH levels and chronic HD vintage [14]. Moreover, PTH levels a couple of times higher than normal levels are considered necessary to maintain normal bone turnover in patients with chronic kidney disease (CKD); low bone responsiveness to PTH is also observed as CKD progresses [15]. Sprague et al. reported the diagnostic accuracy of bone-turnover markers and bone histology in patients on dialysis (almost all the patients were on HD) [16], demonstrating that PTH is still the best tool for the non-invasive assessment of bone turnover in clinical practice; however, TRACP-5b was not assessed in this study.

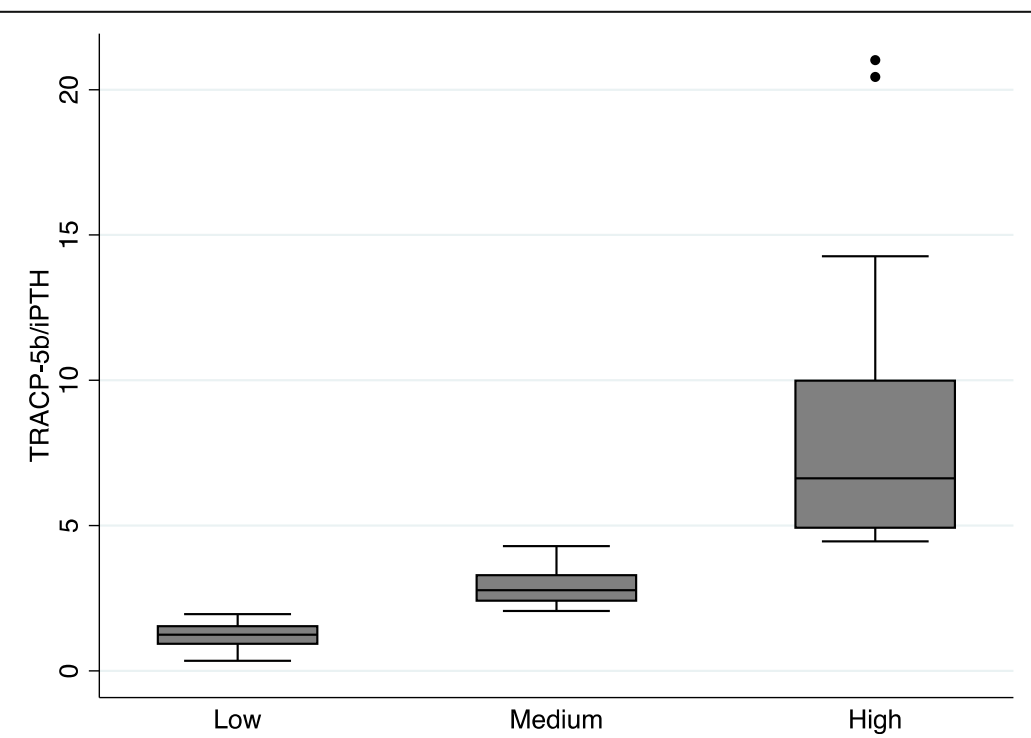

Fig. 1 Box plots of differences in the TRACP-5b/iPTH ratio among the three categories of patients. Abbreviations: iPTH, intact parathyroid hormone; TRACP-5b, tartrate-resistant acid phosphatase-5b 
(a)

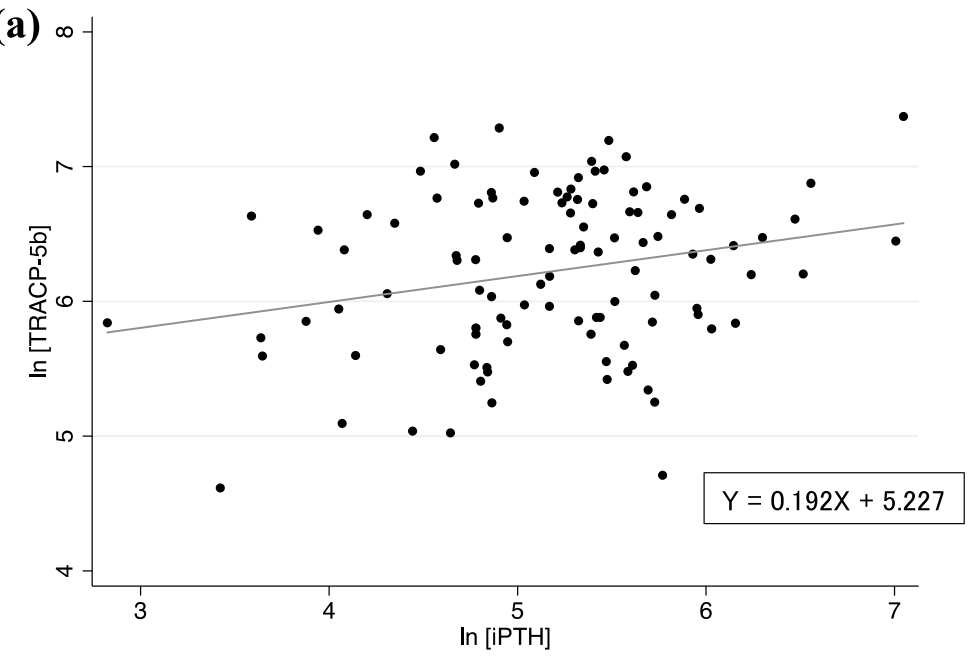

(b)

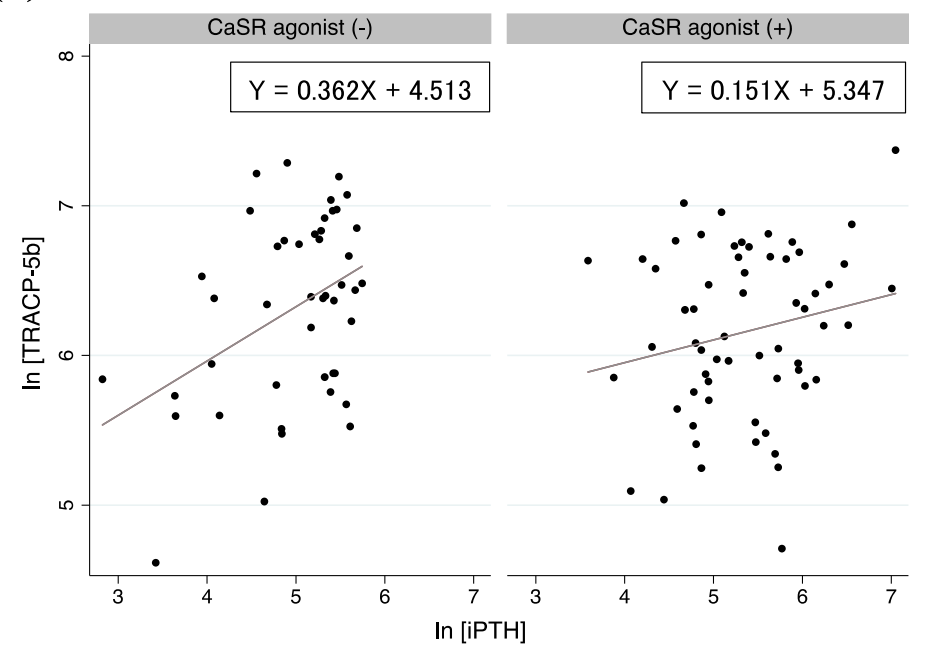

(c)

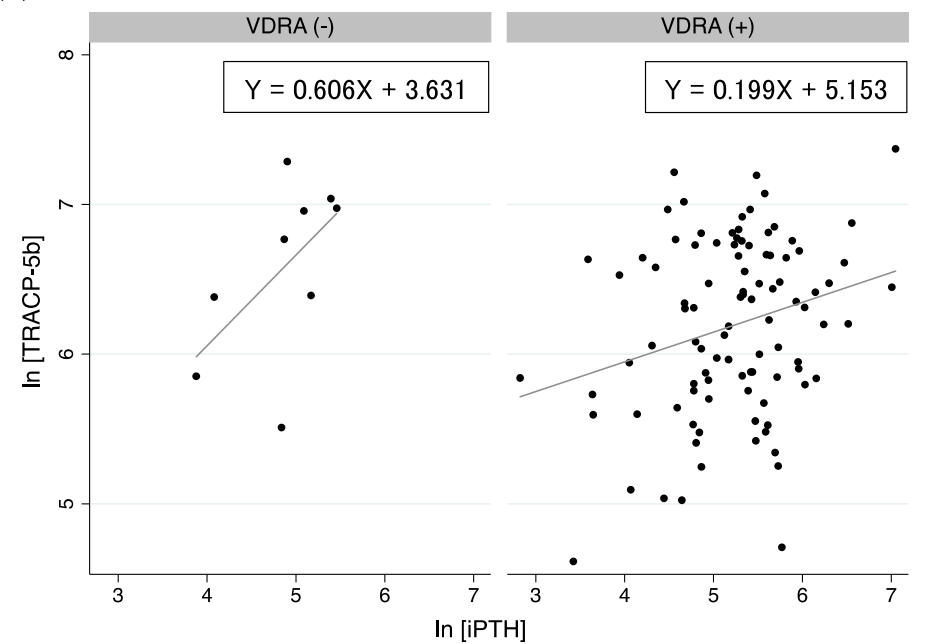

Fig. 2 Scatterplots of $\ln [\mathrm{iPTH}]$ with In [TRACP-5b] with linear regression prediction lines. The plots depict results in a all patients, $\mathbf{b}$ patients receiving the CaSR agonist, and c patients receiving the VDRA. Abbreviations: CaSR, calcium-sensing receptor; iPTH, intact parathyroid hormone; In, natural logarithm; TRACP-5b, tartrate-resistant acid phosphatase-5b; VDRA, vitamin D receptor activator 
Table 3 Single linear regression analysis for In [TRACP-5b/iPTH]

\begin{tabular}{|c|c|c|c|}
\hline Variable & Coefficient & $P$ value & $95 \% \mathrm{Cl}$ \\
\hline Age, years & 0.018 & 0.016 & $0.003,0.032$ \\
\hline Female sex & 0.152 & 0.353 & $-0.171,0.476$ \\
\hline $\mathrm{BMI}, \mathrm{kg} / \mathrm{m}^{2}$ & -0.042 & 0.026 & $-0.080,-0.005$ \\
\hline Chronic HD vintage, years & -0.005 & 0.639 & $-0.027,0.017$ \\
\hline \multicolumn{4}{|l|}{ Anticoagulant during $\mathrm{HD}$} \\
\hline Heparin & (base) & (base) & (base) \\
\hline LMWH & -0.359 & 0.194 & $-0.905,0.186$ \\
\hline Nafamostat & 0.373 & 0.287 & $-0.317,1.063$ \\
\hline \multicolumn{4}{|l|}{ Cause of ESRD } \\
\hline Diabetic nephropathy & (base) & (base) & (base) \\
\hline Nephrosclerosis & -0.041 & 0.854 & $-0.479,0.397$ \\
\hline Glomerulonephritis & -0.046 & 0.831 & $-0.470,0.379$ \\
\hline Others & 0.156 & 0.516 & $-0.320,0.633$ \\
\hline Diabetes & -0.096 & 0.569 & $-0.431,0.278$ \\
\hline Ischemic heart disease & -0.085 & 0.647 & $-0.453,0.283$ \\
\hline Cerebrovascular disease & -0.294 & 0.171 & $-0.718,0.129$ \\
\hline CaSR agonist use & -0.532 & 0.001 & $-0.840,-0.225$ \\
\hline None & (base) & (base) & (base) \\
\hline Cinacalcet/ Evocalcet & -0.450 & 0.004 & $-0.757,-0.143$ \\
\hline Etelcalcetide (i.v.) & -1.290 & $<0.001$ & $-1.953,-0.625$ \\
\hline VDRA use & -0.744 & 0.009 & $-1.301,-0.187$ \\
\hline None & (base) & (base) & (base) \\
\hline Alfacalcidol/ Falecalcitriol & -0.467 & 0.098 & $-1.021,0.087$ \\
\hline Calcitriol (i.v.) & -1.013 & 0.001 & $-1.588,-0.437$ \\
\hline Maxacalcitol (i.v.) & -1.021 & 0.002 & $-1.650,-0.392$ \\
\hline$P$ binder use & -0.041 & 0.856 & $-0.490,0.407$ \\
\hline None & (base) & (base) & (base) \\
\hline Ca-containing & 0.278 & 0.380 & $-0.348,0.905$ \\
\hline Non-Ca containing & -0.166 & 0.482 & $-0.632,0.300$ \\
\hline Both & 0.090 & 0.739 & $-0.444,0.624$ \\
\hline Steroid use history & -0.212 & 0.391 & $-0.699,0.276$ \\
\hline$\beta$ blocker use & -0.165 & 0.310 & $-0.486,0.156$ \\
\hline Statin use & -0.129 & 0.483 & $-0.492,0.234$ \\
\hline Warfarin use & 0.432 & 0.184 & $-0.209,1.073$ \\
\hline Bisphosphonate use & -0.120 & 0.615 & $-0.594,0.353$ \\
\hline Fracture history & 0.185 & 0.427 & $-0.274,0.644$ \\
\hline Ankle-brachial index & -0.115 & 0.831 & -1.176 .0 .947 \\
\hline Alb, g/dL & -0.251 & 0.344 & $-0.774,0.272$ \\
\hline $\mathrm{cCa}, \mathrm{mg} / \mathrm{dL}$ & 0.319 & 0.007 & 0.090 .0 .548 \\
\hline $\mathrm{P}, \mathrm{mg} / \mathrm{dL}$ & -0.173 & 0.018 & $-0.316,-0.031$ \\
\hline $\ln [25(\mathrm{OH}) \mathrm{D}]$ & -0.275 & 0.208 & $-0.705,0.155$ \\
\hline $\ln \left[1,25(\mathrm{OH})_{2} \mathrm{D}\right]$ & 0.250 & 0.044 & $0.007,0.493$ \\
\hline In [BAP] & 0.195 & 0.282 & $-0.163,0.553$ \\
\hline
\end{tabular}

Abbreviations: Alb Serum albumin, BAP Bone-specific alkaline phosphatase, BMI Body mass index, CaSR Calcium-sensing receptor, $c C a$ Corrected Ca, ESRD End-stage renal disease, HD Hemodialysis, iPTH Intact parathyroid hormone, i.v. Intravenous, LMWH Low-molecular-weight heparin, In Natural logarithm, $P$ Phosphorus, TRACP-5b Tartrate-resistant acid phosphatase-5b, VDRA Vitamin D receptor activator 
Table 4 Multiple linear regression analysis for In [TRACP-5b/iPTH]

\begin{tabular}{|c|c|c|c|c|}
\hline Variable & Model 1 & Model 2 & Model 3 & Model 4 \\
\hline Age, years & & $0.006[-0.008,0.021]$ & $0.004[-0.011,0.019]$ & $0.007[-0.009,0.023]$ \\
\hline Female sex & & $0.204[-0.086,0.494]$ & $0.186[-0.107,0.478]$ & $0.190[-0.103,0.484]$ \\
\hline $\mathrm{BMI}, \mathrm{kg} / \mathrm{m}^{2}$ & & & $-0.019[-0.057,0.019]$ & $-0.020[-0.057,0.018]$ \\
\hline Alb, g/dL & & & & $0.219[-0.321,0.759]$ \\
\hline \multicolumn{5}{|l|}{ CaSR agonist use } \\
\hline None & (base) & (base) & (base) & (base) \\
\hline Cinacalcet/ Evocalcet & $-0.329^{*}[-0.642,-0.015]$ & $-0.313[-0.630,0.004]$ & $-0.296[-0.623,0.030]$ & $-0.284[-0.612,0.045]$ \\
\hline Etelcalcetide (i.v.) & $-1.029^{* *}[-1.716,-0.342]$ & $-0.932^{* *}[-1.629,-0.236]$ & $-0.940^{* *}[-1.639,-0.240]$ & $-0.886^{*}[-1.599,-0.173]$ \\
\hline \multicolumn{5}{|l|}{ VDRA use } \\
\hline None & (base) & (base) & (base) & (base) \\
\hline Alfacalcidol/ Falecalcitriol & $-0.349[-0.926,0.236]$ & $-0.344[-0.927,0.239]$ & $-0.299[-0.888,0.290]$ & $-0.305[-0.895,0.285]$ \\
\hline Calcitriol (i.v.) & $-0.770^{* *}[-1.340,-0.200]$ & $-0.815^{* *}[-1.389,-0.240]$ & $-0.772^{*}[-1.353,-0.191]$ & $-0.791^{* *}[-1.374,-0.207]$ \\
\hline Maxacalcitol (i.v.) & $-0.749^{*}[-1.385,-0.113]$ & $-0.791^{*}[-1.434,-0.148]$ & $-0.810^{*}[-1.456,-0.164]$ & $-0.831^{*}[-1.481,-0.182]$ \\
\hline $\mathrm{cCa}, \mathrm{mg} / \mathrm{dL}$ & $0.171[-0.057,0.399]$ & $0.117[-0.057,0.398]$ & $0.177[-0.051,0.406]$ & $0.209[-0.033,0.451]$ \\
\hline$P, \mathrm{mg} / \mathrm{dL}$ & $-0.123[-0.264,0.017]$ & $-0.113[-0.257,0.029]$ & $-0.095[-0.245,0.054]$ & $-0.097[-0.247,0.053]$ \\
\hline $\ln \left[1,25(\mathrm{OH})_{2} \mathrm{D}\right]$ & $-0.156[-0.470,0.158]$ & $-0.207[-0.528,0.113]$ & $-0.207[-0.529,0.115]$ & $-0.213[-0.535,0.110]$ \\
\hline
\end{tabular}

Abbreviations: Alb Serum albumin, BMI Body mass index, CaSR Calcium-sensing receptor, cCa Corrected Ca, iPTH Intact parathyroid hormone, i.v. Intravenous, In Natural logarithm, $P$ Phosphorus, TRACP-5b Tartrate-resistant acid phosphatase-5b, VDRA Vitamin D receptor activator

Values are expressed as the median [interquartile range (IQR)] or as frequencies and percentages [n $(\%)] .{ }^{*} P<0.05,{ }^{* *} P<0.01$ Coefficient $[95 \% \mathrm{Cl}]$

Model 1: CaSR agonist use (None, Cinacalcet/Evocalcet, Etelcalcetide), VDRA use (None, Alfacalcidol/ Falecalcitriol, Calcitriol, Maxacalcitol), cCa, P, In [1, 25(OH) 2 D] Model 2: CaSR agonist use (None, Cinacalcet/Evocalcet, Etelcalcetide), VDRA use (None, Alfacalcidol/ Falecalcitriol, Calcitriol, Maxacalcitol), cCa, P, In [1, 25(OH) 2 D], Age, Sex

Model 3: CaSR agonist use (None, Cinacalcet/Evocalcet, Etelcalcetide), VDRA use (None, Alfacalcidol/ Falecalcitriol, Calcitriol, Maxacalcitol), cCa, P, In [1, 25(OH) 2 D], Age, Sex, BMI

Model 4: CaSR agonist use (None, Cinacalcet/Evocalcet, Etelcalcetide), VDRA use (None, Alfacalcidol/ Falecalcitriol, Calcitriol, Maxacalcitol), cCa, P, In [1, 25(OH) 2 D], Age, Sex, BMI, Alb

The serum TRACP-5b activity is correlated with bone resorption marker levels and osteoclast numbers [17, 18]. Although literature on TRACP-5b in patients on dialysis is scarce, the TRACP-5b level was found to be correlated with most histomorphometric and histodynamic parameters [19]. Additionally, TRACP-5b is the only bone resorption marker not cleared by the kidneys and is an ideal marker for assessing bone resorption in patients on chronic HD [20]. The intra-individual coefficient of variation of TRACP-5b in patients receiving chronic HD is quite low (8.3\%) [21].

Table 5 Multiple linear regression analysis on mineral metabolism for In [TRACP-5b/iPTH]

\begin{tabular}{llll}
\hline Variable & Coefficient & P value & $\mathbf{9 5 \% ~ C l}$ \\
\hline Age & 0.009 & 0.256 & $-0.007,0.025$ \\
BMl & -0.026 & 0.180 & $-0.065,0.012$ \\
CCa & 0.243 & 0.044 & $0.007,0.480$ \\
P & -0.080 & 0.307 & $-0.235,0.075$ \\
In $\left[1,25(\mathrm{OH})_{2} \mathrm{D}\right]$ & 0.078 & 0.563 & $-0.190,0.347$ \\
\hline
\end{tabular}

Abbreviations: BMI Body mass index, cCa Corrected Ca, In Natural logarithm, $P$ Phosphorus
For the above reasons, to investigate bone responsiveness to PTH, we retrospectively assessed the TRACP$5 \mathrm{~b} / \mathrm{iPTH}$ ratio in patients receiving chronic HD. In the present study, the median patient age was 68 years, $42.5 \%$ patients were female (all postmenopausal), 35.8\% had type 2 diabetes, and diabetic nephropathy was the cause of ESRD in approximately one-third of the patients (Table 1). In the laboratory tests, the median serum $\mathrm{cCa}, \mathrm{P}$, and plasma iPTH levels were $9.1 \mathrm{mg} / \mathrm{dL}$, $5.4 \mathrm{mg} / \mathrm{dL}$, and $199.1 \mathrm{pg} / \mathrm{mL}$, respectively, all of which were in the recommended ranges based on the Japanese CKD-MBD guideline [12]. The median TRACP-5b level was $559 \mathrm{mU} / \mathrm{dL}$ (Table 1). Including patients with CKD, bone responsiveness to $\mathrm{PTH}$ increases in the following order: men < premenopausal women < postmenopausal women [22]. In the present study, there was no significant difference in the percentage of female patients among the three categories (Table 2). Although bone responsiveness to PTH decreases in patients on chronic $\mathrm{HD}$ and those with diabetes [23], the percentage of patients with diabetes was not significantly different among the three categories (Table 2). The median serum $1,25(\mathrm{OH})_{2} \mathrm{D}$ level was significantly different among the three TRACP-5b/iPTH categories (Table 2). Disturbed 
mineral metabolism, i.e., higher serum $\mathrm{cCa}$ and $\mathrm{P}$ and lower serum $1,25(\mathrm{OH})_{2} \mathrm{D}$ levels, was associated with reduced bone responsiveness to PTH in the single linear regression analyses. In an additional multiple linear regression analysis performed to uncover the association between mineral metabolism and $\ln$ [TRACP-5b/iPTH] only using the variables identified as significant in the single linear regression analyses (age, $\mathrm{BMI}$, serum $\mathrm{cCa}, \mathrm{P}$ levels, and $\left.\ln \left[1,25(\mathrm{OH})_{2} \mathrm{D}\right]\right)$, only the serum $\mathrm{cCa}$ level exhibited a significant positive association with $\ln$ [TRACP-5b/iPTH] (Table 5). However, from a clinical point of view, higher serum $\mathrm{P}$ and lower serum $1,25(\mathrm{OH})_{2} \mathrm{D}$ levels could lead to higher plasma iPTH levels, resulting in lower TRACP-5b/iPTH ratios. Moreover, the decreased bone responsiveness to PTH in younger patients could be because higher $\mathrm{P}$ intake can lead to a high PTH level, resulting in a lower TRACP$5 \mathrm{~b} / \mathrm{iPTH}$ ratio. On the contrary, in postmenopausal and/ or lean older patients with low protein intake and/or exercise, serum TRACP-5b levels might increase, leading to a higher TRACP-5b/iPTH ratio.

In clinical practice, we prescribe a CaSR agonist and/ or a VDRA to decrease high iPTH levels; therefore, the iPTH level might be related to the serum $1,25(\mathrm{OH})_{2} \mathrm{D}$ level in each category. Interestingly, the types of CaSR agonists and VDRAs used were significantly different among the three categories (Table 2). Upon conducting multiple linear regression analysis incorporating variables identified as significant in the single linear regression analysis (Table 3), a significant negative correlation was found between the TRACP-5b/iPTH ratio and the intravenous administration of a CaSR agonist (etelcalcetide) and/or a VDRA (calcitriol or maxacalcitol) in all the adjusted models (Table 4).

Some reports mention the potential effects of CaSR agonists on bone responsiveness to $\mathrm{PTH}$. CaSR agonists may directly affect bone cells because CaSR is expressed on bone tissue [24]. Activation of CaSR in osteocytes and a high extracellular $\mathrm{Ca}$ level seem to exert osteogenic effects by inducing osteoblast differentiation and osteoclast apoptosis [25]. It has been reported that strontium, a calcimimetic, stimulates CaSR, promotes the differentiation of preosteoblasts into osteoblasts, promotes bone formation, and inhibits the action of osteoclast differentiation factors by secreting osteoprotegerin (OPG) from osteoblasts, which can lead to the inhibition of bone resorption by preventing the RANKL-mediated differentiation of preosteoblasts into osteoclasts [26]. Díaz-Tocados et al. [27] first reported that AMG 641, an allosteric CaSR agonist, exerted a direct anabolic effect on the bone through activation of the CaSR in bone cells of a uremic rat model. In nephrectomized rats with parathyroidectomy and $\mathrm{PTH}$ infusion, the CaSR agonist increased the bone turnover at low PTH levels but not at high PTH levels, suggesting that SHPT reduces the direct effect of CaSR agonists on the bone. Since CaSR agonists and VDRAs were administered to our patients with SHPT, the reduced bone responsiveness to PTH observed in these patients (indicated by a steep coefficient in Fig. 2b and c) is consistent with the results reported previously.

In the participating centers of the present study, the choice of a CaSR agonist for the treatment for SHPT mainly depended on (1) whether patients could take it orally, (2) the degree of gastrointestinal symptoms caused by an oral CaSR agonist (cinacalcet), and (3) whether high PTH levels could be controlled by an oral CaSR agonist, in this order. In the present study, only the use of etelcalcetide showed a significant negative correlation with the TRACP-5b/iPTH ratio among CaSR agonists, although the number of patients receiving etelcalcetide was small (Table 4). Etelcalcetide, a synthetic peptide CaSR agonist, is the first intravenous formulation with a mechanism of action similar to that of cinacalcet [28]; etelcalcetide was found to be superior to cinacalcet in the reduction of iPTH levels among patients on chronic HD with SHPT with iPTH levels greater than $500 \mathrm{pg} / \mathrm{mL}$ [29]. Notably, etelcalcetide and cinacalcet show differences in terms of pharmacokinetics. Since the half-life of cinacalcet is approximately 30 $\mathrm{h}, \mathrm{PTH}$ levels in patients receiving cinacalcet show oscillations [30]. However, the half-life of etelcalcetide is as long as 3-5 days [31]; thus, etelcalcetide keeps PTH secretion suppressed and may not cause PTH level oscillations in patients with SHPT. This difference in pharmacokinetics among CaSR agonists may also influence bone responsiveness to PTH.

The introduction of intravenous calcitriol was associated with marked reductions in PTH levels and bone turnover [32], and a study reported improvement in high-turnover bone lesions by calcitriol in chronic HD patients [33]. There are also some reports on the potential effects of VDRA on bone responsiveness to PTH. By acting on osteoblasts, VDRA produces OPG, which neutralizes RANKL, suppresses osteoclast production and activity, and finally induces osteoclast apoptosis [34]. There are reports indicating that calcitriol downregulates $\mathrm{PTH} / \mathrm{PTHrP}$ receptor gene expression in osteoblast-like cells [35] and that intracellular cAMP levels increase in response to $\mathrm{PTH}$ in osteoblasts, but this increase is suppressed by treatment with $1,25(\mathrm{OH})_{2} \mathrm{D}$ [36]. Kikuta et al. reported that VDRAs significantly suppress the expression of sphingosine-1-phosphate receptor 2 in circulating osteoclasts and inhibit bone resorption by osteoclasts through monocyte progenitor cells migrating from the bone to blood [37].

In addition to the use of the intravenous CaSR agonist in the present study, only the use of the intravenous 
VDRAs exhibited a significant negative correlation with the TRACP-5b/iPTH level among VDRAs (Table 4). In a prospective randomized study, the effects of oral and intravenous pulse calcitriol on serum levels of IL-1 beta and IL- 6 and bone-resorptive cytokines implicated for changes in bone remodeling were compared in patients on chronic HD [38]. Despite there being no difference in serum PTH levels after 6 months, intravenous calcitriol therapy caused a greater increase in bone mineral densities and a greater decrease in serum IL- 6 levels than oral calcitriol therapy; this suggested that compared to oral therapy, intravenous calcitriol treatment exerted a superior effect on bone remodeling by influencing the levels of bone-resorptive cytokines, beyond its suppressive effect on $\mathrm{iPTH}$. The iPTH assay was used to measure the total length of $\mathrm{PTH}$, consisting of 84 amino groups, simultaneously including a large $\mathrm{C}-\mathrm{PTH}$ fragment, such as PTH (7-84) (cyclase inhibitory PTH, CIP) [39]. Furthermore, PTH (7-84) was shown to have an antagonistic effect on PTH (1-84) (cyclase activating $\mathrm{PTH}, \mathrm{CAP}$ ) [38]. Of note, intravenous VDRA treatment was shown to reduce the PTH (1-84)/large C fragments ratio in patients on chronic HD [40], which could reduce bone responsiveness to PTH. Indeed, maxacalcitol is as effective as calcitriol in suppressing PTH in patients on chronic HD [41], but a report demonstrated that intravenous maxacalcitol therapy in chronic HD patients markedly improved bone histology, without a decrease in PTH levels [42].

The present study has several strengths. This is the first report on the association between CaSR agonist and/or VDRA use and bone responsiveness to PTH in a chronic HD population. In clinical practice, the commonly utilized bone-turnover markers in CKD are BAP and $\mathrm{iPTH}$; however, we used TRACP-5b as a specific biomarker of osteoclast number and activity, as it is not influenced by renal function and its level was reported to be correlated with most histomorphometric and histodynamic parameters [19]. We also used the means of six consecutive values of plasma iPTH levels measured once per month, prior to measuring the serum TRACP$5 \mathrm{~b}$ level, to avoid influence by a single iPTH level. We created the TRACP-5b/iPTH ratio as an index of bone responsiveness to $\mathrm{PTH}$. Indeed, the major mechanisms underlying the lowering of PTH levels are the inhibition of PTH secretion by CaSR agonists and the suppression of PTH synthesis by VDRAs in the parathyroid glands. However, the present study suggests that HD patients with low TRACP-5b/iPTH ratios are representative of those with bone resistance (decreased bone responsiveness) to PTH, probably associated with severe SHPT, which requires treatment with CaSR agonists and/or VDRAs, as reported by Díaz-Tocados et al. [27]. Therefore, the TRACP-5b/iPTH ratio could serve as an index of the bone responsiveness to $\mathrm{PTH}$ in patients on chronic HD in clinical practice.

Several limitations should also be considered while interpreting or generalizing the present findings. First, this was a retrospective, cross-sectional study that used limited information available in each patient's electronic health record; thus, we could not confirm a causal relationship between the intravenous use of a CaSR agonist and/or VDRA and bone responsiveness to PTH expressed by the TRACP-5b/iPTH ratio. In fact, CaSR agonists and/or VDRAs are prescribed to lower high iPTH levels in clinical practice, which means that there might or might not be differences in the backgrounds of patients subjected to intravenous administration of the two agents. Second, we have addressed neither the dosing period nor the dosage of these drugs. Third, there is a lack of data regarding whole PTH, FGF-23 levels, and bone histomorphometry; thus, the findings of this study do not support further speculation regarding the causal relationship between the intravenous use of a CaSR agonist and/or VDRA and bone responsiveness to PTH. Fourth, the number of patients included in the analysis was relatively small, implying that the statistical significance of the results was low. Finally, this study was conducted only in Japanese SHPT patients undergoing chronic HD whose iPTH levels were controlled to be lower than those of patients in other regions, based on the Japanese CKD-MBD guideline [12].

\section{Conclusions}

The present study indicates that bone responsiveness to PTH not only varies but also is negatively associated with the intravenous administration of CaSR agonists and/or VDRAs in patients undergoing chronic HD.

\section{Abbreviations \\ SHPT: Secondary hyperparathyroidism; HD: Hemodialysis; PTH: Parathyroid hormone; RANKL: Receptor activator of nuclear factor-kappa B ligand; TRACP- 5b: Tartrate-resistant acid phosphatase 5b; CaSR: Calcium-sensing receptor; VDRA: Vitamin D receptor activator; iPTH: Intact parathyroid hormone; IRB: Institutional Review Board; Alb: Albumin; Ca: Calcium; P: Phosphorus; BAP: Bone-specific alkaline phosphatase; 25(OH)D: 25-hydroxyvitamin D; 1,25(OH) 2 D: 1,25-dihydroxycholecalciferol; cCa: Corrected calcium; CLEIA: Chemiluminescent enzyme immunoassay; EIA: Enzyme immunoassay; ECLIA: Electrochemiluminescence assay; CLIA: Chemiluminescence assay; RIA: Radioimmunoassay; ABI: Ankle-brachial index; BMI: Body mass index; IQR: Interquartile range; Cl: Confidence interval; In: Natural logarithm; i.v.: Intravenously; KDIGO: the Kidney Disease: Improving Global Outcomes; JSDT: The Japanese Society for Dialysis Therapy; CKD: Chronic kidney disease; OPG: Osteoprotegerin; CIP: Cyclase inhibitory PTH; CAP: Cyclase activating PTH}

\section{Acknowledgments}

Portions of this work were presented at the Annual Meeting of the American Society of Nephrology in Washington, DC in November 2019. The authors thank Mayumi Kudo and Haruna Ishikawa, pharmacists of the Kidney Center of Kawasaki Municipal Tama Hospital and Dr. Tomohiro Yonaha, Honorary Director of Yonaha Medical Clinic, for managing the data for this study. We also thank Editage (www.editage.jp) for their English language editing service. 


\section{Authors' contributions}

Naoto Tominaga conceived this study, collected and interpreted data, performed statistical analyses, and drafted and revised the manuscript. Tomoki Yonaha collected data. Masayuki Yamanouchi, Hirofumi Sumi, and Yasuhiro Taki provided intellectual content of critical importance. Yugo Shibagaki, Kazuhiro Shiizaki, and Shozo Yano interpreted the data, provided intellectual content of critical importance, and revised the manuscript. All authors have approved the final version of the manuscript and agree with its submission to BMC Nephrology.

\section{Funding}

English proofreading of the present manuscript was funded by Mitsubishi Tanabe Pharma (MTPS20200522060).

\section{Availability of data and materials}

The datasets used and/or analyzed in the present study are available from the corresponding author on reasonable request.

\section{Declarations}

\section{Ethics approval and consent to participate}

All procedures performed in this study involving human participants were in accordance with the ethical standards of the Institutional Committee on Human Research of St. Marianna University School of Medicine [the Institutional Review Board (IRB) at St. Marianna University School of Medicine (IRB approval number: 4291)] and with the 1964 Helsinki declaration and its later amendments or comparable ethical standards. Informed consent was waived by our IRB, as the study was retrospective and non-interventional in nature and was based on chart reviews.

\section{Consent for publication}

Not applicable.

\section{Competing interests}

There are no financial or other potential conflicts of interest to disclose regarding this study.

\section{Author details}

'Division of Nephrology and Hypertension, Department of Internal Medicine, St. Marianna University School of Medicine, 2-16-1, Sugao, Miyamae-ku, Kawasaki, Kanagawa 216-8511, Japan. ${ }^{2}$ Division of Nephrology and Hypertension, Kawasaki Municipal Tama Hospital, Kawasaki, Kanagawa, Japan. ${ }^{3}$ Nephrology and Dialysis Center, Yohana Medical Clinic, Ishigaki, Okinawa, Japan. ${ }^{4}$ Nephrology Center, Toranomon Hospital Kajigaya, Kawasaki, Kanagawa, Japan. ${ }^{5}$ Department of Nephrology, Yurina Medical Park, Nogi, Tochigi, Japan. ${ }^{6}$ Department of Laboratory Medicine, Shimane University Faculty of Medicine, Izumo, Shimane, Japan.

Received: 8 February 2021 Accepted: 23 July 2021

Published online: 09 August 2021

\section{References}

1. Jadoul M, Albert JM, Akiba T, Akizawa T, Arab L, Bragg-Gresham JL, et al. Incidence and risk factors for hip or other bone fractures among hemodialysis patients in the Dialysis outcomes and practice patterns study. Kidney Int. 2006;70(7):1358-66. https://doi.org/10.1038/s.ki.5001754.

2. Tentori F, McCullough K, Kilpatrick RD, Bradbury BD, Robinson BM, Kerr PG, et al. High rates of death and hospitalization follow bone fracture among hemodialysis patients. Kidney Int. 2014;85(1):166-73. https://doi.org/10.1038/ ki.2013.279.

3. Kalantar-Zadeh K, Miller JE, Kovesdy CP, Mehrotra R, Lukowsky LR, Streja E, et al. Impact of race on hyperparathyroidism, mineral disarrays, administered vitamin D mimetic, and survival in hemodialysis patients. J Bone Miner Res. 2010;25(12):2724-34. https://doi.org/10.1002/jbmr.177.

4. Silva BC, Bilezikian JP. Parathyroid hormone: anabolic and catabolic actions on the skeleton. Curr Opin Pharmacol. 2015;22:41-50. https://doi.org/10.101 6/j.coph.2015.03.005

5. Henriksen K, Tanko LB, Qvist P, Delmas PD, Christiansen C, Karsdal MA. Assessment of osteoclast number and function: application in the development of new and improved treatment modalities for bone diseases. Osteoporos Int. 2007;18(5):681-5. https://doi.org/10.1007/s00198-006-0286-8.
6. Behets GJ, Spasovski G, Sterling LR, Goodman WG, Spiegel DM, De Broe ME, et al. Bone histomorphometry before and after long-term treatment with cinacalcet in dialysis patients with secondary hyperparathyroidism. Kidney Int. 2015;87(4):846-56. https://doi.org/10.1038/ki.2014.349.

7. Payne RB, Little AJ, Williams RB, Milner JR. Interpretation of serum calcium in patients with abnormal serum proteins. Br Med J. 1973;4(5893):643-6. https://doi.org/10.1136/bmj.4.5893.643.

8. Fukagawa M, Shimazaki R, Akizawa T, Evocalcet study group. Head-to-head comparison of the new calcimimetic agent evocalcet with cinacalcet in Japanese hemodialysis patients with secondary hyperparathyroidism. Kidney Int. 2018;94(4):818-25. https://doi.org/10.1016/j.kint.2018.05.013.

9. London GM, Marchais SJ, Guérin AP, de Vernejoul MC. Ankle-brachial index and bone turnover in patients on dialysis. J Am Soc Nephrol. 2015 Feb; 26(2):476-83. https://doi.org/10.1681/ASN.2014020169.

10. Tentori F, Blayney MJ, Albert JM, Gillespie BW, Kerr PG, Bommer J, et al. Mortality risk for dialysis patients with different levels of serum calcium, phosphorus, and PTH: the Dialysis outcomes and practice patterns study (DOPPS). Am J Kidney Dis. 2008;52(3):519-30. https://doi.org/10.1053/j.ajkd.2 008.03.020.

11. Kidney Disease: Improving Global Outcomes (KDIGO) CKD-MBD Work Group. KDIGO clinical practice guideline for the diagnosis, evaluation, prevention, and treatment of Chronic Kidney Disease-Mineral and Bone Disorder (CKD-MBD). Kidney Int Suppl. 2009;113:S1-130.

12. Fukagawa M, Yokoyama K, Koiwa F, Taniguchi M, Shoji T, Kazama JJ, et al. Japanese Society for Dialysis Therapy. Clinical practice guideline for the management of chronic kidney disease-mineral and bone disorder. Ther Apher Dial. 2013;17(3):247-88. https://doi.org/10.1111/1744-9987.12058.

13. National Kidney Foundation. K/DOQI clinical practice guidelines for bone metabolism and disease in chronic kidney disease. Am J Kidney Dis. 2003; 42(4 Suppl 3):S1-201.

14. Nickolas TL, Stein EM, Dworakowski E, Nishiyama KK, Komandah-Kosseh M, Zhang $\mathrm{CA}$, et al. Rapid cortical bone loss in patients with chronic kidney disease. J Bone Miner Res. 2013;28(8):1811-20. https://doi.org/10.1002/jbmr.1916.

15. Imasaki Y, Yamato H, Nii-Kono T, Fujieda A, Uchida M, Hosokawa A, et al. Insufficiency of PTH action on bone in uremia. Kidney Int Suppl. 2006;102:S34-6.

16. Sprague SM, Bellorin-Font E, Jorgetti V, Carvalho AB, Malluche HH, Ferreira A, et al. Diagnostic accuracy of bone turnover markers and bone histology in patients with CKD treated by Dialysis. Am J Kidney Dis. 2016;67(4):559-66. https://doi.org/10.1053/j.ajkd.2015.06.023.

17. Halleen JM, Tiitinen SL, Ylipahkala H, Fagerlund KM, Väänänen HK. Tartrateresistant acid phosphatase $5 b$ (TRACP $5 b$ ) as a marker of bone resorption. Clin Lab. 2006;52(9-10):499-509.

18. Janckila AJ, Slone SP, Lear SC, Martin A, Yam LT. Tartrate-resistant acid phosphatase as an immunohistochemical marker for inflammatory macrophages. Am J Clin Pathol. 2007 Apr;127(4):556-66. https://doi.org/1 0.1309/DGEA9BE2VE5VCFYH.

19. Coen G, Ballanti P, Bonucci E, Calabria S, Centorrino M, Fassino V, et al. Bone markers in the diagnosis of low turnover osteodystrophy in haemodialysis patients. Nephrol Dial Transplant. 1998;13(9):2294-302. https://doi.org/10.1 093/ndt/13.9.2294

20. Shidara K, Inaba M, Okuno S, Yamada S, Kumeda Y, Imanishi Y, et al. Serum levels of TRAP5b, a new bone resorption marker unaffected by renal dysfunction, as a useful marker of cortical bone loss in hemodialysis patients. Calcif Tissue Int. 2008;82(4):278-87. https://doi.org/10.1007/s00223008-9127-4.

21. Cavalier E, Delanaye P, Moranne O. Variability of new bone mineral metabolism markers in patients treated with maintenance hemodialysis: implications for clinical decision making. Am J Kidney Dis. 2013;61(5):847-8. https://doi.org/10.1053/j.ajkd.2012.12.013.

22. Kotowicz MA, Klee GG, Kao PC, O'Fallon WM, Hodgson SF, Cedel SL, et al. Relationship between serum intact parathyroid hormone concentrations and bone remodeling in type I osteoporosis: evidence that skeletal sensitivity is increased. Osteoporos Int. 1990;1 (1):14-22. https://doi.org/10.1 007/BF01880411.

23. Yoshida O, Inaba M, Terada M, Shioi A, Nishizawa Y, Otani S, et al. Impaired response of human osteosarcoma (MG-63) cells to human parathyroid hormone induced by sustained exposure to high glucose. Miner Electrolyte Metab. 1995;21(1-3):201-4.

24. Brown EM, MacLeod RJ. Extracellular calcium sensing and extracellular calcium signaling. Physiol Rev. 2001;81(1):239-97. https://doi.org/10.1152/ physrev.2001.81.1.239. 
25. Kanatani M, Sugimoto T, Kanzawa M, Yano S, Chihara K. High extracellular calcium inhibits osteoclast-like cell formation by directly acting on the calciumsensing receptor existing in osteoclast precursor cells. Biochem Biophys Res Commun. 1999;261(1):144-8. https://doi.org/10.1006/bbrc.1999.0932.

26. Hamdy NA. Strontium ranelate improves bone microarchitecture in osteoporosis. Rheumatology (Oxford). 2009:48(Suppl 4):iv9-13.

27. Díaz-Tocados JM, Rodríguez-Ortiz ME, Almadén Y, Pineda C, Martínez-Moreno $J M$, Herencia C, et al. Calcimimetics maintain bone turnover in uremic rats despite the concomitant decrease in parathyroid hormone concentration. Kidney Int. 2019;95(5):1064-78. https://doi.org/10.1016/j.kint.2018.12.015.

28. Walter S, Baruch A, Dong J, Tomlinson JE, Alexander ST, Janes J, et al. Pharmacology of AMG 416 (Velcalcetide), a novel peptide agonist of the calcium-sensing receptor, for the treatment of secondary hyperparathyroidism in hemodialysis patients. J Pharmacol Exp Ther. 2013; 346(2):229-40. https://doi.org/10.1124/jpet.113.204834.

29. Block GA, Bushinsky DA, Cheng S, Cunningham J, Dehmel B, Drueke TB, et al. Effect of Etelcalcetide vs Cinacalcet on serum parathyroid hormone in patients receiving hemodialysis with secondary hyperparathyroidism: a randomized clinical trial. JAMA. 2017;317(2):156-64. https://doi.org/10.1001/jama.2016.19468.

30. Padhi D, Harris R. Clinical pharmacokinetic and pharmacodynamic profile of cinacalcet hydrochloride. Clin Pharmacokinet. 2009:48(5):303-11. https://doi. org/10.2165/00003088-200948050-00002.

31. Wu B, Melhem M, Subramanian $R$, Chen $P$, Jaramilla Sloey B, Fouqueray $B$, et al. Clinical pharmacokinetics and pharmacodynamics of Etelcalcetide, a novel Calcimimetic for treatment of secondary hyperparathyroidism in patients with chronic kidney Disease on hemodialysis. J Clin Pharmacol. 2018;58(6):717-26. https://doi.org/10.1002/jcph.1090.

32. Slatopolsky E, Weerts C, Thielan J, Horst R, Harter H, Martin KJ. Marked suppression of secondary hyperparathyroidism by intravenous administration of 1,25-dihydroxy-cholecalciferol in uremic patients. J Clin Invest. 1984;74(6):2136-43. https://doi.org/10.1172/JCl111639.

33. Baker LR, Muir JW, Sharman VL, Abrams SM, Greenwood RN, Cattell WR, et al. Controlled trial of calcitriol in hemodialysis patients. Clin Nephrol. 1986;26(4):185-91.

34. Hofbauer LC, Dunstan CR, Spelsberg TC, Riggs BL, Khosla S. Osteoprotegerin production by human osteoblast lineage cells is stimulated by vitamin $D$, bone morphogenetic protein-2, and cytokines. Biochem Biophys Res Commun. 1998;250(3):776-81. https://doi.org/10.1006/bbrc.1998.9394.

35. González EA, Martin KJ. Coordinate regulation of PTH/PTHrP receptors by PTH and calcitriol in UMR 106-01 osteoblast-like cells. Kidney Int. 1996;50(1): 63-70. https://doi.org/10.1038/ki.1996.287.

36. Wong GL, Luben RA, Cohn DV. 1,25-dihydroxycholecalciferol and parathormone: effects on isolated osteoclast-like and osteoblast-like cells. Science. 1977;197(4304):663-5. https://doi.org/10.1126/science.195343.

37. Kikuta J, Kawamura S, Okiji F, Shirazaki M, Sakai S, Saito H, et al. Sphingosine-1-phosphate-mediated osteoclast precursor monocyte migration is a critical point of control in antibone-resorptive action of active vitamin D. Proc Natl Acad Sci U S A. 2013;110(17):7009-13. https://doi.org/1 0.1073/pnas.1218799110

38. Türk S, Akbulut M, Yildiz A, Gürbilek M, Gönen S, Tombul Z, et al. Comparative effect of oral pulse and intravenous calcitriol treatment in hemodialysis patients: the effect on serum IL-1 and IL-6 levels and bone mineral density. Nephron. 2002;90(2):188-94. https://doi.org/10.1159/00004 9041.

39. Slatopolsky E, Finch J, Clay P, Martin D, Sicard G, Singer G, et al. A novel mechanism for skeletal resistance in uremia. Kidney Int. 2000;58(2):753-61. https://doi.org/10.1016/S0085-2538(15)47156-X.

40. Kihara T, Ichikawa H, Morimoto H, Yano A, Akagi S, Nakao K, et al. Intravenous vitamin D therapy reduces PTH-(1-84)/large C fragments ratio in chronic hemodialysis patients. Nephron Clin Pract. 2004;98(3):C93-100. https://doi.org/10.1159/000080680.

41. Mizobuchi M, Ogata H. Clinical uses of 22-oxacalcitriol. Curr Vasc Pharmacol. 2014;12(2):324-8. https://doi.org/10.2174/15701611113119990023.

42. Tsukamoto Y, Hanaoka M, Matsuo T, Saruta T, Nomura M, Takahashi Y. Effect of 22-oxacalcitriol on bone histology of hemodialyzed patients with severe secondary hyperparathyroidism. Am J Kidney Dis. 2000;35(3):458-64. https:// doi.org/10.1016/S0272-6386(00)70198-3

\section{Publisher's Note}

Springer Nature remains neutral with regard to jurisdictional claims in published maps and institutional affiliations.

\section{Ready to submit your research? Choose BMC and benefit from}

- fast, convenient online submission

- thorough peer review by experienced researchers in your field

- rapid publication on acceptance

- support for research data, including large and complex data types

- gold Open Access which fosters wider collaboration and increased citations

- maximum visibility for your research: over $100 \mathrm{M}$ website views per year

At $\mathrm{BMC}$, research is always in progress.

Learn more biomedcentral.com/submissions 